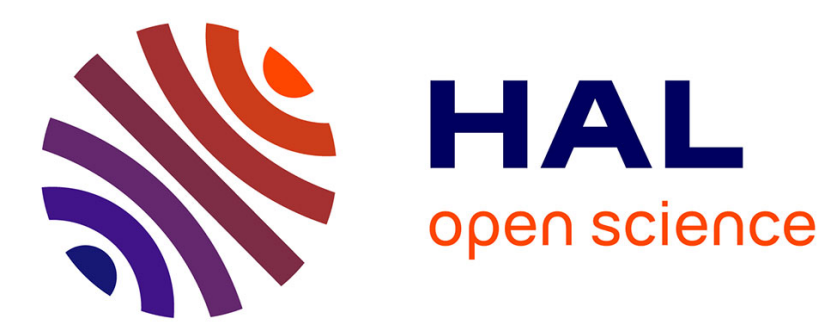

\title{
Methods for Gibbs triple junction excess determination: Ti segregation in CoSi2 thin film
}

Hannes Zschiesche, Ahmed Charai, Claude Alfonso, Dominique Mangelinck

\section{To cite this version:}

Hannes Zschiesche, Ahmed Charai, Claude Alfonso, Dominique Mangelinck. Methods for Gibbs triple junction excess determination: Ti segregation in CoSi2 thin film. Journal of Materials Science, 2020, 55 (27), pp.13177-13192. 10.1007/s10853-020-04856-4 . hal-03060639

\section{HAL Id: hal-03060639 \\ https://hal.science/hal-03060639}

Submitted on 23 Dec 2020

HAL is a multi-disciplinary open access archive for the deposit and dissemination of scientific research documents, whether they are published or not. The documents may come from teaching and research institutions in France or abroad, or from public or private research centers.
L'archive ouverte pluridisciplinaire HAL, est destinée au dépôt et à la diffusion de documents scientifiques de niveau recherche, publiés ou non, émanant des établissements d'enseignement et de recherche français ou étrangers, des laboratoires publics ou privés. 


\title{
Methods for Gibbs triple junction excess determination - Ti segregation in $\mathrm{CoSi}_{2}$ thin film
}

\author{
Hannes Zschiesche ${ }^{\mathrm{a}, *}$, Ahmed Charai ${ }^{\mathrm{a}}$, Claude Alfonso ${ }^{\mathrm{a}}$, Dominique \\ Mangelinck ${ }^{\mathrm{b}}$ \\ ${ }^{a}$ Aix-Marseille Université, CNRS, IM2NP, Faculté de Saint-Jérôme, Marseille, France \\ ${ }^{b}$ CNRS, Aix-Marseille Université, IM2NP, Faculté de Saint-Jérôme, Marseille, France
}

\begin{abstract}
Methods are presented determining the Gibbs triple junction excess $\left(\Gamma^{\mathrm{TJ}}\right)$ of solute segregation in poly crystalline materials from single atom counting in $3 \mathrm{D}$ volumes. One method bases on cumulative profile analysis while two further methods use radial integration of solute atoms. The methods are demonstrated and compared on simulated model volumes which include three grain boundaries joining together at a triple junction with set values for Gibbs grain boundary and triple junction excess. An experimental technique that provides $3 \mathrm{D}$ volumes with single atom detection and spatial resolution close to atomic scale is atom probe tomography. An atom probe tomography volume of a $\mathrm{CoSi}_{2}$ thin film that contains three grain boundaries and a triple junction has been acquired. $\mathrm{Ti}$ segregation is found qualitatively at the grain boundaries and triple junction. The quantification of the $\mathrm{Ti}$ excess at the investigated $\mathrm{CoSi}_{2}$ triple junction reveals for the three introduced methods positive Gibbs triple junction excess values. It demonstrates that there is an excess of $\mathrm{Ti}$ at $\mathrm{CoSi}_{2}$ triple junctions and provides opportunities for its quantification.
\end{abstract}

Keywords: Gibbs excess, Triple junction, Atom probe tomography, Ti segregation, $\mathrm{CoSi}_{2}$ thin film

\section{Introduction}

Triple junctions (TJs) are present in poly crystalline material where three grain boundaries (GBs) join together. They influence directly the thermodynamics and kinetics of materials and thereby their properties. Thus, their de5 scription by geometric and thermodynamic parameters is of great interest 1-

\footnotetext{
${ }^{*}$ Corresponding author: Hannes Zschiesche

Email address: hannes.zschiesche@im2np.fr (Hannes Zschiesche )
} 
4. Experimental measurements of TJ energies were demonstrated using Scanning Tunneling Microscopy [5] or Atom Force Microscopy [6]. Simulations have brought insides to the view on TJ energies from the theoretical approach [7, 8].

Segregation of solutes to TJs can also change the energy of the TJ [9]. Direct measurements of TJ segregation were obtained by energy dispersive x-ray spectroscopy (EDS) 10, 11. Thickness measurements by energy filtered transmission electron microscopy (EFTEM) have allowed to conclude the presence of cavities in the TJ region [12 that was interpreted as a segregation of vacancies. However, these kind of TEM based measurements are limited through 2D projections. In contrast, atom probe tomography (APT) allows 3D measurements at the atomic scale 13, 14]. For example, measurements on a thermally treated $\mathrm{Ni}-\mathrm{Cu}$ bilayer system have demonstrated that there is a significantly higher $\mathrm{Ni}$ diffusion in $\mathrm{Cu}$ TJs than in $\mathrm{Cu}$ GB [15]. Further studies on the same material system have revealed that additionally there is a lower activation energy for 20 TJ diffusion than for GB diffusion [16, 17. Similar investigations on thermally treated Fe-Cr multilayers have determined a TJ segregation enthalpy of $\mathrm{Cr}$ in Fe TJ [18, 19. These experimental results have shown qualitatively some excess at TJ. Moreover, quantification of the excess at a TJ was obtained recently from a theoretical approach that studied GB and TJ segregation in the Fe-Cr 25 system [20]. However, for a quantitative comparison between experimental and theoretical data, methods are required for determination of the Gibbs TJ excess $\left(\Gamma^{T J}\right.$ ) 21] by APT similarly to the established method for Gibbs GB excess $\left(\Gamma^{G B}\right)$ 22].

TJs are of particular importance for poly crystalline thin films, because their 30 proportion is larger due to small grain size. Especially, this is true in silicide thin films that are used in microelectronics. $\mathrm{CoSi}_{2}$ films on $\mathrm{Si}$ substrates are widely used in microelectronics as contact material beside $\mathrm{TiSi}_{2}$ and $\mathrm{NiSi}$ films, 
depending on the scale. Low electrical resistivity, high thermal stability and the good match of the crystal structure with $\mathrm{Si}$ are the main advantages of

$35 \mathrm{CoSi}_{2}$ 23]. The reaction of thin cobalt films with silicon substrates leads to sequential formation of $\mathrm{Co}_{2} \mathrm{Si}, \mathrm{CoSi}$ and $\mathrm{CoSi}_{2}$ [24, 25]. Ti was introduced as an interlayer between the Co layer and the Si substrate to promote a strong texture (epitaxy) in $\mathrm{CoSi}_{2}$ grown on $\mathrm{Si}(100)$ or $\mathrm{Si}(111)$ by sputtering deposition and reactive diffusion [26]. The role of the Ti interlayer during the phase formation

40 has been investigated and explanations were proposed, such as the reduction of native oxide at the Si surface [27 29], the formation of a diffusion barrier for Co [27, 29, 30] and the variation of $\mathrm{CoSi}_{2}$ nucleation due to Ti segregation at the Si interface [31. The latter one was proven by the influence of a $\mathrm{Ti}$ capping layer instead of a Ti interlayer [32, 33. The Ti capping layer induced

45 an increase of the nucleation temperature of $\mathrm{CoSi}_{2}$ as well as a promoted texture in comparison to the growth without capping Ti. These effects were explained by the diffusion of $\mathrm{Ti}$ from the capping layer to the interface. Recently, the presence of Ti segregation at $\mathrm{CoSi}_{2}$ GBs has been shown [34].

In this work, Ti segregation at $\mathrm{CoSi}_{2}$ TJs is studied. For a quantitative determination of $\Gamma^{T J}$, three methods are developed. They are compared on a model volume with known excess values, before application on an experimentally acquired volume.

\section{Material and Methods}

$\mathrm{CoSi}_{2}$ thin films were grown on $\mathrm{Si}(111)$ wafers which were immersed in a $555 \%$ dilute $\mathrm{HF}$ for 1 min to remove native oxide before load into the sputtering system. Successive deposition of $5 \mathrm{~nm}$ Ti and $100 \mathrm{~nm}$ Co from two individual targets of $\mathrm{Ti}$ and $\mathrm{Co}$ (both $99.99 \%$ purity) was done by magnetron sputtering. The deposition was performed with a base pressure of about $10^{-8}$ Torr using 
$99.9999 \%$ pure Ar and a gas flow of $6.7 \mathrm{sccm}$ at room temperature. Thermal

60 deposited sample into $\mathrm{CoSi}_{2}$.

APT tips were prepared using a focused ion beam (FIB) FEI Helios 600 with a $\mathrm{Ga}^{+}$source [35, 36]. APT measurements were performed on a LEAP 3000X HR. Voltage mode at $40 \mathrm{~K}, 200 \mathrm{kHz}, 20 \%$ pulse fraction and $0.2 \%$ detection rate was applied in order to get optimal information as a compromise between resolution and volume size. IVAS software was used for the reconstruction following the common procedure [37, 38. The reconstruction parameters were adjusted with crystallographic information: they were changed in order to match the plane spacing obtained from the observed planes belonging to a pole

\section{Results}

Firstly, the creation of a model volume is described. This model volume contains 3 grains, 3 GBs and the TJ with different solute contents. Then, the methods developed to quantify Gibbs TJ excess are demonstrated on such a model system, before an experimentally acquired volume is presented and $\mathrm{Ti}$ excess at the present $\mathrm{CoSi}_{2}$ TJ is quantified.

\subsection{Simulation of model volume with known excess values}

To test the quantification methods of this Gibbs TJ excess, model volumes were simulated using Matlab. The parameters where chosen close to the ones observed in an experimental volume in order to give a realistic representation. Fig. 1a shows a schematic projection of the described geometry including the geometrical parameters in the projected planes. A cube with $\mathrm{CoSi}_{2}$ structure (fcc, $\mathrm{CaF}_{2}$ type) with a single orientation, i.e. a single crystal, was built in 


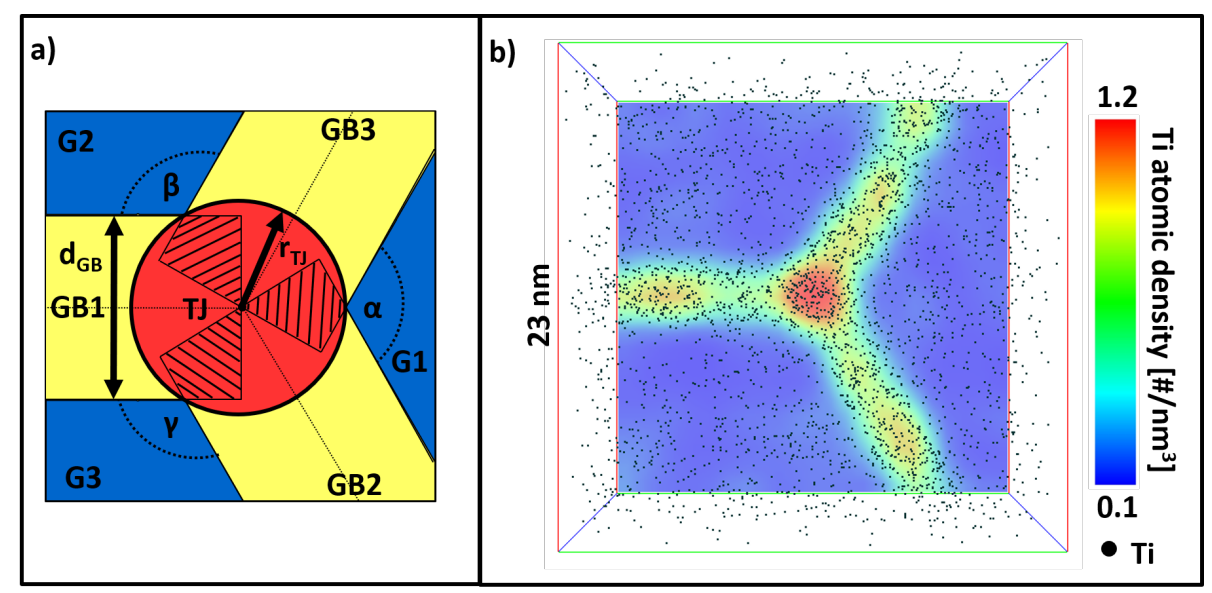

Figure 1: a) Geometries used in the simulated model volume for a projected view along TJ and GBs. b) Simulated model volume (cube) with Gibbs GB and TJ excess together with a projected $2 \mathrm{D} \mathrm{Ti}$ atomic density map that visualizes qualitatively the excess at GBs and TJ (set values: $\rho_{\mathrm{Ti}}^{\mathrm{G}}=0.18 \mathrm{at} / \mathrm{nm}^{3}, \rho_{\mathrm{Ti}}^{\mathrm{GB}}=0.51 \mathrm{at} / \mathrm{nm}^{3}, \rho_{\mathrm{Ti}}^{\mathrm{TJ}}=0.94 \mathrm{at} / \mathrm{nm}^{3} ; d_{\mathrm{GB}}=3 \mathrm{~nm}$; $\left.\Gamma_{\mathrm{Ti}}^{\mathrm{GB}, 1}=\Gamma_{\mathrm{Ti}}^{\mathrm{GB}, 2}=\Gamma_{\mathrm{Ti}}^{\mathrm{GB}, 3}=1 \mathrm{at} / \mathrm{nm}^{2} ; \Gamma_{\mathrm{Ti}}^{\mathrm{TJ}}=4 \mathrm{at} / \mathrm{nm}\right)$.

order to simulate a 3D volume with atomic resolution. In this volume, a given

number of Ti atoms per unit volume (atomic density) were introduced such as:

$$
\rho_{\mathrm{Ti}}^{\mathrm{G}}=\frac{N_{\mathrm{Ti}}^{\mathrm{G}}}{V_{\mathrm{G}}}=\frac{x_{\mathrm{Ti}}^{\mathrm{G}}}{\omega_{\mathrm{CoSi} 2}}
$$

where $N_{\mathrm{Ti}}^{\mathrm{G}}$ is the number of Ti atoms in the considered grain volume $V_{\mathrm{G}}, x_{\mathrm{Ti}}^{\mathrm{G}}$ is the atomic fraction and $\omega_{\mathrm{CoSi} 2}$ the atomic volume of $\mathrm{CoSi}_{2}$. In this definition, the atomic density of $\mathrm{Ti}$ is directly linked to the atomic fraction of Ti since the addition of $\mathrm{Ti}$ is assumed to not modify the atomic volume of $\mathrm{CoSi}_{2}$ (i.e. $\mathrm{Ti}$ substitutes either $\mathrm{Si}$ or Co and does not modify the lattice parameter of $\mathrm{CoSi}_{2}$ ). Based on this approximation, $\rho_{\mathrm{Ti}}^{\mathrm{G}}$ was generated by random substitution of a fraction $x_{\mathrm{Ti}}^{\mathrm{G}}$ of Co and $\mathrm{Si}$ atoms by Ti atoms. It was further assumed that the Ti density was the same in the different grains since it should be the case at equilibrium.

The three GBs inside this cube are defined as rectangular slice volumes 
that go from the border of the cubic volume to its center (Fig. 117). They have all the same width $d_{\mathrm{GB}}$ and they share a same direction corresponding to the TJ axis. In the limit that the GB width goes to zero $\left(d_{\mathrm{GB}} \rightarrow 0\right)$, the GB describes a plane that separates two grains. Note that the grains in our simulated volume are just defined as regions delimited by GBs and do not have different orientations between them in contrary to the real case where their orientation is different. The Gibbs GB excess $\left(\Gamma^{\mathrm{GB}}\right)$ [21] of segregated Ti is given in atoms per area of GB. By assuming that the Ti distribution is uniform inside the GB slice, the Gibbs GB excess can be transformed to an Ti atomic density inside the rectangular slice volume by dividing through $d_{\mathrm{GB}}$. Adding the $\mathrm{Ti}$ atomic density of the neighboring grains results in the total $\mathrm{Ti}$ atomic density inside the defined GB volume.

$$
\rho_{\mathrm{Ti}}^{\mathrm{GB}}=\rho_{\mathrm{Ti}}^{\mathrm{G}}+\frac{\Gamma_{\mathrm{Ti}}^{\mathrm{GB}}}{d_{\mathrm{GB}}}=\rho_{\mathrm{Ti}}^{\mathrm{G}}+\frac{N_{\mathrm{Ti}}^{\mathrm{GB}, \mathrm{Exc}}}{V_{\mathrm{GB}}}=\frac{N_{\mathrm{Ti}}^{\mathrm{GB}}}{V_{\mathrm{GB}}}=\frac{x_{\mathrm{Ti}}^{\mathrm{GB}}}{\omega_{\mathrm{CoSi} 2}}
$$

There is thus an additional (excess) number of Ti atoms $\left(N_{\mathrm{Ti}}^{\mathrm{GB}, \mathrm{Exc}}\right)$ inside the GB volume $\left(V_{\mathrm{GB}}\right)$ in comparison to the grains. As a uniform atomic density is assumed in the GB, the Gibbs GB excess is realized by random substitution of the fraction $x_{\mathrm{Ti}}^{\mathrm{GB}}$ of Co or Si by Ti in the GB volume.

The TJ is defined as a cylindrical volume with a radius $r_{\mathrm{TJ}}$ along the center line of the cube (Fig. 1 1 ). $r_{\mathrm{TJ}}$ was geometrically fixed as the minimal cylinder radius which includes all GB overlap at the TJ (hatched regions in Fig. 1p). ${ }_{115}$ It was thus determined from the largest distance of a GB overlap to the TJ center (Eq. A.2 in Appendix A which depends on the dihedral angle between adjacent $\mathrm{GBs}(\alpha, \beta, \gamma)$ and the GB width $\left(d_{\mathrm{GB}}\right)$. In the limit that the GB width goes to zero $\left(d_{\mathrm{GB}} \rightarrow 0\right)$, the TJ radius goes to zero $\left(r_{\mathrm{TJ}} \rightarrow 0\right)$ and the TJ represents the junction line of the three GBs. Similarly to the Gibbs GB excess, the Gibbs TJ excess of Ti segregated at the TJ $\left(\Gamma^{\mathrm{TJ}}\right)$ is given in atoms per 
length on such a line. A transformation of $\Gamma^{\mathrm{TJ}}$ to a number per 3D unit volume (i.e. a Ti atomic density) can be achieved by dividing through the base area of the TJ cylinder volume. The total Ti atomic density inside this TJ volume depends on the atomic densities of the bordering grains, the atomic density of the GB excess respecting their volume fraction inside the TJ volume $\left(f_{\mathrm{GB}, \mathrm{i}}^{\mathrm{TJ}}\right.$, Eq. A.4 in Appendix A and the atomic density related to the TJ excess. Note that to calcualte the GB fraction in the TJ cylinder volume $\left(f_{\mathrm{GB}, \mathrm{i}}^{\mathrm{TJ}}\right)$, one has to consider the overlap (hatched regions in Fig. 17) in order to avoid an artificial TJ excess (Eq. A.3 in Appendix A.

$$
\begin{aligned}
\rho_{\mathrm{Ti}}^{\mathrm{TJ}} & =\rho_{\mathrm{Ti}}^{\mathrm{G}}+\sum_{i=1}^{3} f_{\mathrm{GB}, \mathrm{i}}^{\mathrm{TJ}} \cdot \frac{\Gamma_{\mathrm{Ti}}^{\mathrm{GB}, \mathrm{i}}}{d_{\mathrm{GB}, \mathrm{i}}}+\frac{\Gamma_{\mathrm{Ti}}^{\mathrm{TJ}}}{\pi \cdot r_{\mathrm{TJ}}^{2}} \\
& =\rho_{\mathrm{Ti}}^{\mathrm{G}}+\sum_{i=1}^{3} f_{\mathrm{GB}, \mathrm{i}}^{\mathrm{TJ}} \cdot \frac{\Gamma_{\mathrm{Ti}}^{\mathrm{GB}, \mathrm{i}}}{d_{\mathrm{GB}, \mathrm{i}}}+\frac{N_{\mathrm{Ti}}^{\mathrm{TJ}} \mathrm{Exc}}{V_{\mathrm{TJ}}}=\frac{N_{\mathrm{Ti}}^{\mathrm{TJ}}}{V_{\mathrm{TJ}}}=\frac{x_{\mathrm{Ti}}^{\mathrm{TJ}}}{\omega_{\mathrm{CoSi} 2}}
\end{aligned}
$$

A uniform distribution is also assumed in the TJ and the number of $\mathrm{Ti}$ atoms $\left(N_{\mathrm{Ti}}^{\mathrm{TJ}}\right)$ inside the $\mathrm{TJ}$ volume $\left(V_{\mathrm{TJ}}\right)$ was made by random substitution of a fraction $x_{\mathrm{Ti}}^{T J}$ of Co or $\mathrm{Si}$ by $\mathrm{Ti}$ in the TJ volume.

A simulated model volume with set GB and TJ excess is shown in Fig. 1 $\mathrm{b}$. The $\mathrm{Ti}$ atoms are visible as dark points in the 3D cubic volume (side length $23 \mathrm{~nm})$. A $2 \mathrm{D} \mathrm{Ti}$ atomic density map which is projected along the viewing direction (parallel to the TJ) is also shown in Fig. 1 $1 \mathrm{~b}$. The color scale of the $2 \mathrm{D}$ atomic density map ranges from low Ti atomic density (blue) in grain regions to high Ti atomic density (red) in the TJ region. In this model system, the atomic density for a given atom map is equivalent to an atomic fraction since the atomic density for all atoms (i.e. the inverse of the atomic volume) inside the cube is set to constant in the approximation that there is no change in the atomic volume of $\mathrm{CoSi}_{2}$ (i.e. $0.0128 \mathrm{~nm}^{3} /$ at) when $\mathrm{Ti}$ is introduced substitutionally. The $2 \mathrm{D}$ atomic density map in Fig. 10 is similar to the schematic in Fig. 11. Knowing 
the set values inside the model volume will now permit to test Gibbs TJ excess quantification methods.

\subsection{Gibbs TJ excess quantification methods applied on simulated model volumes}

Three different methods will be applied to simulated model volumes in order to quantify $\Gamma^{\mathrm{TJ}}$. In the simulated model volumes, Ti Gibbs TJ and GB excess values have been set to $\Gamma_{\mathrm{Ti}}^{\mathrm{TJ}}=4 \mathrm{at} / \mathrm{nm}$ and $\Gamma_{\mathrm{Ti}}^{\mathrm{GB}}=1 \mathrm{at} / \mathrm{nm}^{2}$ and the Ti atomic varied from narrow $\operatorname{GBs}\left(d_{\mathrm{GB}}=0.5 \mathrm{~nm}\right)$ to wide $\operatorname{GBs}\left(d_{\mathrm{GB}}=3.0 \mathrm{~nm}\right)$ and equal dihedral angles $\left(\alpha=\beta=\gamma=120^{\circ} \mathrm{C}\right)$. Accordingly to Eq. A.2 $r_{\mathrm{TJ}}$ was set to $0.3 \mathrm{~nm}\left(\right.$ when $\left.d_{\mathrm{GB}}=0.5 \mathrm{~nm}\right)$ and $1.7 \mathrm{~nm}\left(\right.$ when $\left.d_{\mathrm{GB}}=3.0 \mathrm{~nm}\right)$. The values are close to the one observed in an experimental volume (see Sec. 3.3. .

\subsubsection{Cumulative profile method - linear integration}

The method proposed in this paragraph bases on a cumulative profile similarly to the method used for determination of the Gibbs GB excess 22. Therefore, a cuboid volume is centered along a GB crossing the TJ perpendicular (Fig. 2a). The width of the cuboid can be varied (dotted arrows) to optimize the geometry around the TJ in order to get the highest sensitivity to the Gibbs TJ excess in the 1D atomic fraction and cumulative profiles. Fig. $2 \mathrm{~b}$ shows the Ti atomic fraction along the linear integration inside the selected cuboid. A separation of the different features inside the cuboid is possible from varying constant Ti atomic fraction regions. As expected, the highest Ti atomic fraction 165 is observed at the region where the TJ is present. However, the values are not directly related to the atomic fractions inside the labelled features, i.e. GBs and/or TJ, but to the average over the width of the cuboid that can include grains, GBs and TJ at the referring location. Because of such averaging effect, it is difficult to extract the value of the TJ excess from such a $1 \mathrm{D} \mathrm{Ti}$ atomic 

excess: it is achieved by plotting the cumulative number of Ti atoms over the cumulative number of all atoms along the analysis direction (black arrow in Fig. 2a). Fig. 26 shows such a cumulative profile on the simulated model volume with $d_{\mathrm{GB}}=0.5 \mathrm{~nm}$. The slope in the cumulative profile refers directly to the has the highest $\mathrm{Ti}$ atomic fraction due to the TJ excess and thus the largest slope: it can be separated from the regions before and after the TJ that are less rich in Ti. The constant atomic fraction regions (slopes) on the left and on the right of the TJ region can be linearly fitted (green dashed lines). In the Gibbs TJ excess value, $\Gamma^{\mathrm{TJ}}$, is calculated by dividing this number of Ti atoms through the length of the TJ included in the cuboid. The Gibbs TJ excess determined by this method is $\Gamma_{\mathrm{Ti}}^{\mathrm{TJ}}=3.6$ at $/ \mathrm{nm}$ which is slightly lower than the set value of $\Gamma_{\mathrm{Ti}}^{\mathrm{TJ}}=4 \mathrm{at} / \mathrm{nm}$. method. Indeed, as detailed by Jenkins et al. [40, the cumulative method suffers from limitations even in the simple case of GB. Furthermore, there are additional limitations for the TJ. Firstly, there is a high sensitivity of the measured value to the choice of the step location: the step height varies strongly when the position of the step is changed $( \pm 0.6$ at/nm for a variation of the TJ center by $25 \%)$ due to the large difference between $\mathrm{Ti}$ atomic fraction in the regions on the left and 


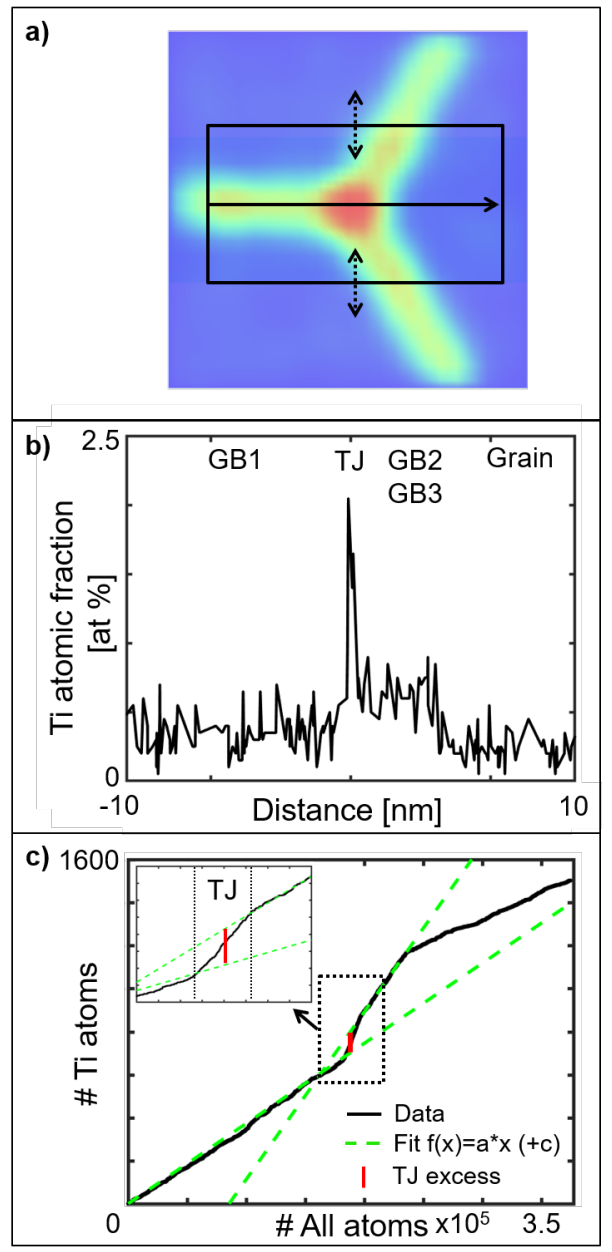

Figure 2: First method to quantify $\Gamma^{\mathrm{TJ}}$. a) A cuboid is defined with two sides in parallel to one GB and the TJ. b) 1D Ti atomic fraction profile shows the different regions of constant atomic fraction: GB1 + grain on the left, GB2 + GB3 + grain on the right of the TJ and pure grain on the outer right. The tight TJ region has the highest Ti atomic fraction. Note that the atomic fraction values do not refer directly to the included features, but to an average inside the selected cuboid at the related integration location. c) A cumulative profile perpendicular to the TJ allows to determine the absolute number of atoms in excess at the TJ from the step height in the plot (inset). $\Gamma^{\mathrm{TJ}}$ is revealed by dividing this step height through the height of the cuboid (length of considered TJ).

on the right of the TJ. This difference comes from the presence of two GBs on the right of the TJ and only one on the left. In addition, the random substitution of Co or Si by Ti can introduce fluctuations in the distribution of Ti. These local 

variations in selected volume, including possible small variations of TJ center selection). In addition, this method is not applicable when the TJ excess is relatively low or the GB width is large since the change corresponding to the TJ region becomes less pronounced. In order to overcome these limitations, two are now considered.

\subsubsection{Radial integration methods}

The second and third methods base on a radial integration of the Ti atoms. In these methods, a cylinder with height $z$ and an increasing radius $r$ is defined with its axis on the TJ line (center line of defined cylindrical TJ region in Fig. 19) and the number of Ti atoms inside the cylinder is counted (as shown schematically in Fig. 3a). The total number of Ti atoms inside the cylinder depends on $r$ as following:

$$
N_{\mathrm{Ti}}(r)=\sum_{i=1}^{3} N_{\mathrm{Ti}}^{\mathrm{G}, \mathrm{i}}(r)+\sum_{j=1}^{3} N_{\mathrm{Ti}}^{\mathrm{GB}, \mathrm{j}, \mathrm{Exc}}(r)+N_{\mathrm{Ti}}^{\mathrm{TJ}, \mathrm{Exc}}(r) .
$$

The contributions of $\mathrm{Ti}$ atoms in the TJ due to the grains, GBs and TJ are Dependencies of the number of $\mathrm{Ti}$ atoms with the radius $r$ are now considered using the previous assumptions (homogeneous atomic densities in the related volumes, same atomic volume in the whole volume,...). While the number of Ti atoms of the grains changes as a square function in $r\left(N_{\mathrm{Ti}}^{\mathrm{G}}(r)=z \cdot \rho_{\mathrm{Ti}}^{\mathrm{G}} \cdot \pi r^{2}\right)$,

the expressions for the GB excess and TJ excess are more complex and two approaches can be distinguished depending on the value of radius: i.e. if $r \gg r_{\mathrm{TJ}}$ (a) or if $r \leq r_{\mathrm{TJ}}$ (b). 
a) When $r \gg r_{\mathrm{TJ}}$, the TJ volume is completely included in the cylinder: the number of Ti atoms related to the TJ excess $\left(N_{\mathrm{Ti}}^{\mathrm{TJ} \text {,Exc }}\right)$ remains thus constant under variation of $r$. In this case, the GB volume inside the cylinder can be approximated as a rectangular slice and the number of $\mathrm{Ti}$ atoms related to the GB excess varies linearly in $r\left(N_{\mathrm{Ti}}^{\mathrm{GB}, \mathrm{Exc}}=z \cdot \Gamma_{\mathrm{Ti}}^{\mathrm{GB}} \cdot r\right)$. The variation of the number of Ti atoms with $\mathrm{r}$ can thus be expressed as

$$
N_{\mathrm{Ti}}(r) / z=\sum_{i} \rho_{\mathrm{Ti}}^{\mathrm{G}, \mathrm{i}} \cdot \pi r^{2}+\sum_{j} \Gamma_{\mathrm{Ti}}^{\mathrm{GB}, \mathrm{j}} \cdot r+\Gamma_{\mathrm{Ti}}^{\mathrm{TJ}}
$$

Fig. 3p shows a radial 1D Ti atomic fraction plot of the simulated model volume used for the cumulative profile method. The Ti atomic fraction is highest in the very tight TJ region (smallest radius $r$ ). Close to the TJ region the atomic fraction is much lower, but not immediately constant since the approximation for Eq. 5does not hold until a certain radius $r$ is reached where the atomic fraction is about constant. This region of about constant atomic fraction is appropriate for the second order polynomial fit and chosen in the radial integral plot. In Fig. 35, the radial integration of $\mathrm{Ti}$ atoms divided through the height of the cylinder (black dots) together with a second order polynomial fit in the region $r \gg r_{\mathrm{TJ}}$ (green dashed line) are shown. The TJ excess is determined as the constant from this second order polynomial fit. The result of $\Gamma_{\mathrm{Ti}}^{\mathrm{TJ}}=3.9 \mathrm{at} / \mathrm{nm}$ is in accordance with the set Gibbs TJ excess in the simulated volume (4 at $/ \mathrm{nm})$.

Nevertheless, a difficulty of this method is that it bases on the constant coefficient of the second order polynom: as this term is usually small compared to other contributions, its value depends critically on the fit quality (variations up to \pm 1.0 at/nm observed for varying fit region). Moreover, if the GB thicknesses is increased, and consequently $r_{\mathrm{TJ}}$, a significant discrepancy between the quantified result and the set value appears. In this case, the approximation of 

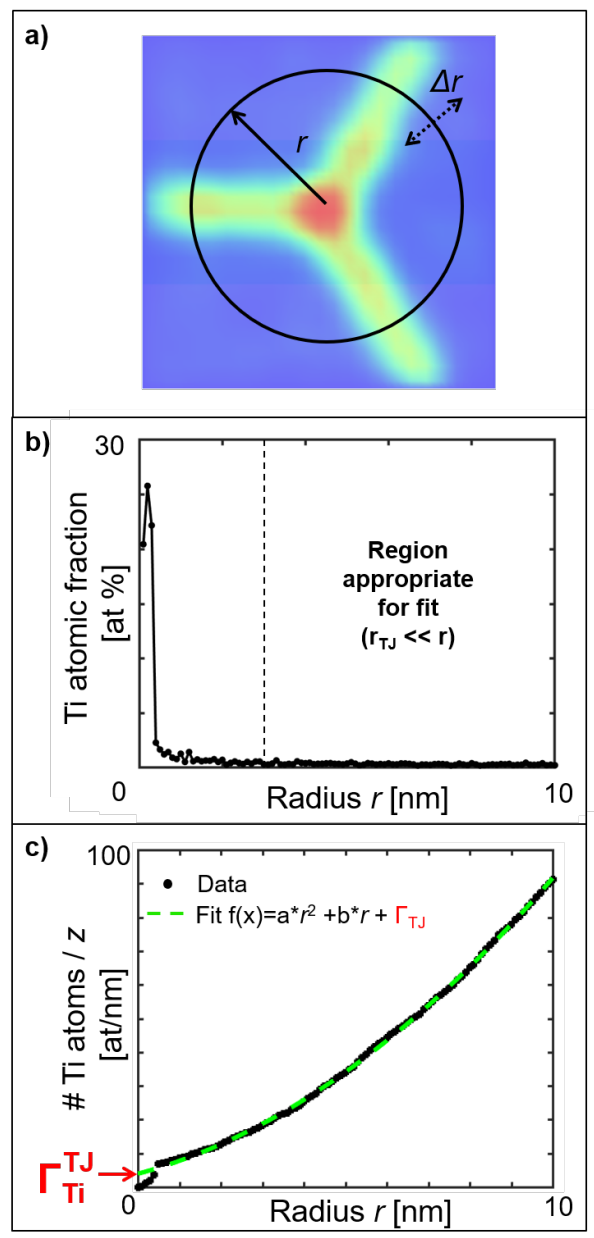

Figure 3: Second method for measureing $\Gamma^{\mathrm{TJ}}$ : Radial integration. a) Defining a cylinder along the $\mathrm{TJ}$ allows a radial integration of the Ti atoms. b) A radial $1 \mathrm{D}$ Ti atomic fraction plot shows the high $\mathrm{Ti}$ atomic fraction inside the TJ region (left), a transition region with much lower and almost constant, but slightly decreasing $\mathrm{Ti}$ atomic fraction and finally a region appropriate for the second order polynomial fit. c) In the approach $r \gg r_{\mathrm{TJ}}$, the contribution of the $\mathrm{Ti}$ atoms in excess at the $\mathrm{TJ}$ is constant (Eq. 5) and determined by a second order polynomial fit.

the GB as a rectangular projection does not hold anymore since the assumption, $r \gg r_{\mathrm{TJ}}$, is not fulfilled and Eq. 5 is no more valid. Therefore, another quantification method is now developed which is applied in the range $r \leq r_{\mathrm{TJ}}$.

b) When $r \leq r_{\mathrm{TJ}}$, the Ti atom number contributions of grain, GBs and TJ 
are proportional to $r^{2}\left(N_{\mathrm{Ti}}(r)=z \cdot \pi r^{2} \cdot \rho_{\mathrm{Ti}}^{\mathrm{TJ}}\right)$ since the Ti atomic density was set as constant in the TJ of the model volume. It means that plotting $N_{\mathrm{Ti}}(r) /(z \cdot r)$ over $r$ should give a linear plot.

$$
N_{\mathrm{Ti}}(r) /(z \cdot r)=\pi \cdot \rho_{\mathrm{Ti}}^{\mathrm{TJ}} \cdot r
$$

255 very close to the set value of $4 \mathrm{at} / \mathrm{nm}$, it is noticed that other created volumes with the same setting configuration can reveal values with higher discrepancy to the TJ excess set value (up to $\pm 1 \mathrm{at} / \mathrm{nm}$ ). This can be due to very limited

Fig. 4a shows the Ti atomic fraction of a model volume with $d_{\mathrm{GB}}=3 \mathrm{~nm}$ in a radial 1D plot binning on equal radius increments. The change of the GB width causes that the TJ region with about constant atomic fraction $\left(r_{\mathrm{TJ}}=1.7 \mathrm{~nm}\right)$ is much broader than the one studied in Fig. $3\left(r_{\mathrm{TJ}}=0.3 \mathrm{~nm}, \mathrm{x}\right.$-scale changed $)$. This broader TJ region is appropriate for an analysis of the TJ atomic density by radial integration in $r \leq r_{\mathrm{TJ}}$.

Fig. $4 \mathrm{~b}$ shows a plot of the integrated number of Ti atoms divided by $z \cdot r$ over $r$. A linear fit (green dashed line) is applied at low cylinder radius $\left(r \leq r_{\mathrm{TJ}}\right)$. Using the set values for the Ti concentration inside the grains, the Gibbs GB excess and the width of the GBs as well as the radius of the TJ volume gives a value for the Gibbs TJ excess of $\Gamma_{\mathrm{Ti}}^{\mathrm{TJ}}=4.1 \mathrm{at} / \mathrm{nm}$. Even though this result is the tJ excess set value (up to $\pm 1 \mathrm{at} / \mathrm{hm}$ ). This can be due to very linited 

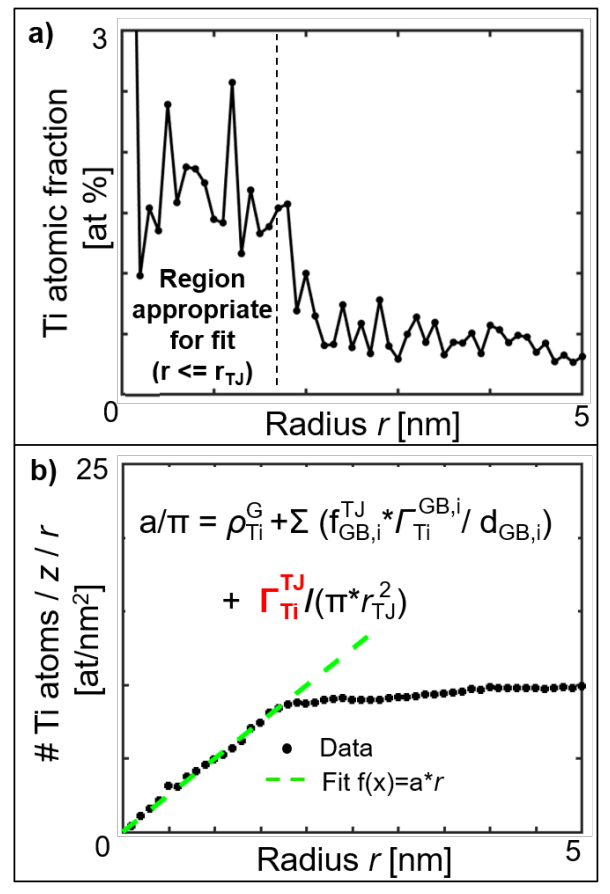

Figure 4: Third method for TJ excess determination: a) Ti atomic fraction in a radial 1D plot equivalent to Fig. 3 , but on the volume with larger GB width $\left(d_{\mathrm{GB}}=3 \mathrm{~nm}\right.$ instead of $d_{\mathrm{GB}}=0.5 \mathrm{~nm}$ ). Note that fluctuations in the atomic fraction from a constant value are present due to low statistics in the radial 1D profile close to the center. b) In the approach $r \leq r_{\mathrm{TJ}}$, the atomic densities of grain, excess at GBs and TJ contribute to a linear slope when the number of Ti atoms per $z$ divided by $r$ is plotted over $r . \Gamma^{\mathrm{TJ}}$ is revealed from this slope and independent determinations of Ti atomic density in grains, GBs and the geometries of GBs and TJ.

statistics inside the selected cylinder. Thereby, small variations in the Ti atom distribution inside the TJ volume can have significant effects on the linear fit and thus on the determined Gibbs TJ excess value.

After the exploration of the methods on model volumes in this section, an application on an experimental volume is of interest.

\subsection{Qualitative Ti excess at experimentally acquired $\mathrm{CoSi}_{2} \mathrm{TJ}$}

Fig. 5a shows a reconstructed APT volume of the $\mathrm{CoSi}_{2}$ thin film containing three different $\mathrm{CoSi}_{2}$ grains. No Ga contamination is detected in the investigated region of interest that could modify the composition of the volume. 
Ti segregation at GBs and TJ makes the three $\mathrm{CoSi}_{2}$ GBs and the $\mathrm{CoSi}_{2} \mathrm{TJ}$ visible. Variation of crystallographic pole location across the GBs were also

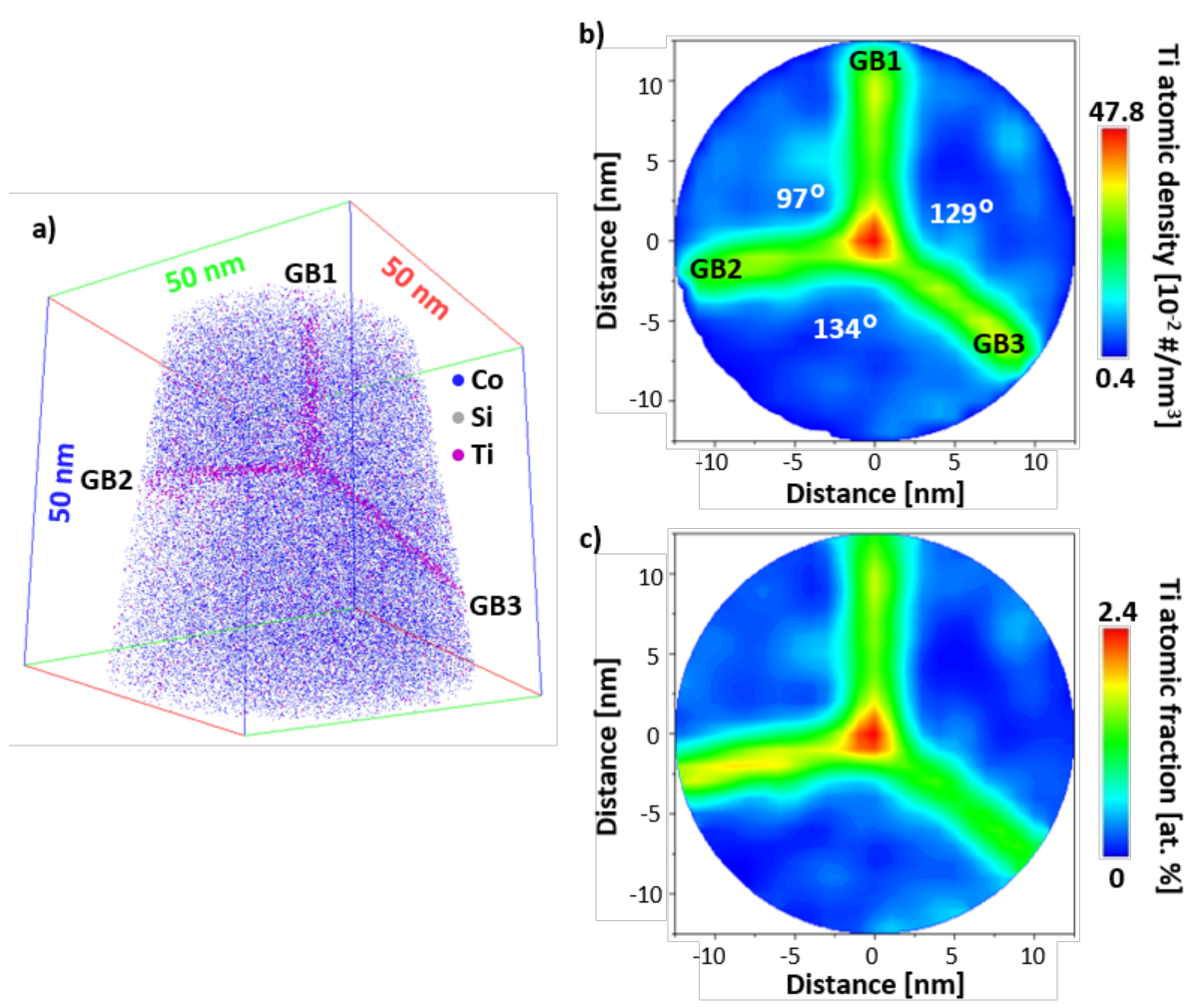

observed from isodensity surfaces (not shown). The angles between the GBs are $\alpha=134^{\circ}, \beta=129^{\circ}$ and $\gamma=97^{\circ}$. These angles between the GB planes are roughly around $120^{\circ}$ that corresponds to a state of equilibrium for GBs with the same interface energies. The Ti atomic fraction inside the $\mathrm{CoSi}_{2}$ grains is measured to be $x_{\mathrm{Ti}}^{\mathrm{G}}=0.2$ at $\%$. The width of the GBs were measured to be about $d_{\mathrm{GB}}=2 \mathrm{~nm}$ and the radius of the TJ volume is thus around $r_{\mathrm{TJ}}=1.2 \mathrm{~nm}$.

Figure 5: a) 3D APT volume of $\mathrm{CoSi}_{2}$ thin film including three GBs and a TJ which show $\mathrm{Ti}$ segregation. b) 2D Ti atomic density map projected along $\mathrm{TJ}$ visualizes qualitatively $\mathrm{Ti}$ excess at GBs and TJ. c) 2D Ti atomic fraction map of same region of interest.

A qualitative view on the Ti excess at the GBs and at the TJ is given in a colored 2D Ti atomic density map projected along the TJ (Fig. 5b). Grain 
regions are in blue, because the $\mathrm{Ti}$ atomic density is low in comparison to the ones of GBs where excess of $\mathrm{Ti}$ is present (green to yellow). The projected GB

295 and parallel to the viewing direction (minimum of $d_{\mathrm{GB}}$ ). Notably, the highest $\mathrm{Ti}$ atomic density in the colored $2 \mathrm{D}$ map is visible at the $\mathrm{TJ}$ region (red). The $\mathrm{Ti}$ atomic density at the $\mathrm{TJ}$ region is two orders of magnitude higher than the $\mathrm{Ti}$ atomic density inside the grains as the atomic densities selected for the simulated model volume. However, the atomic density in tomographic reconstructions can vary artificially due to local magnification and detection efficiency effects when crossing interfaces. The atomic density can also depend on the methodology used to establish the 2D map (sampling volume, vortex, size,...). Consequently, the atomic density obtained from the $2 \mathrm{D}$ map are only semi quantitative and more precise methods should be used.

For the GB excess, the cumulative profile analysis 22] allows an accurate determination: the Gibbs GB excess were determined to $\Gamma_{\mathrm{Ti}}^{\mathrm{GB} 1}=0.9$ at $/ \mathrm{nm}^{2}$, $\Gamma_{\mathrm{Ti}}^{\mathrm{GB} 2}=0.8$ at $/ \mathrm{nm}^{2}$ and $\Gamma_{\mathrm{Ti}}^{\mathrm{GB} 3}=0.8$ at $/ \mathrm{nm}^{2}$ by this method.

Another visualization of segregation is a 2D atomic fraction map (Fig. 5F). 310 Again, the Ti atomic fraction is lowest in grain regions, increased in the GBs and highest in the TJ region. Thus, an additional Ti segregation to the TJ is observed in the 2D maps that requires more precise quantification.

\subsection{Ti Gibbs TJ excess quantification at experimentally measured $\mathrm{CoSi}_{2}$ TJ}

For the quantification of the Ti Gibbs TJ excess at the experimentally acquired $\mathrm{CoSi}_{2}$ TJ, the methods introduced in section 3.2 were applied and the corresponding plots are shown in Fig 6 .

Fig. 6a shows a cumulative profile (black line) along GB3 with the complementary linear 1D Ti atomic fraction plot in Fig. 6d. The TJ region can be 
dotted lines) that refers to the highest atomic fraction in the plot of Fig. 6 d.

It is much less pronounced than in the model system (Fig. 2). The visibility of these variations in the cumulative profile (Fig. 6a) is optimal for a cuboid width between $8 \mathrm{~nm}$ and $11 \mathrm{~nm}$. From the step (red vertical line) at the center of the TJ region between the linear fits (green dashed lines) of the regions at the left and the right of the TJ region, a Ti Gibbs TJ excess is determined to $\Gamma_{\mathrm{Ti}}^{\mathrm{TJ}}=2.2 \mathrm{at} / \mathrm{nm}$.

Plots of the radial integration methods are shown in Fig. 6b and 6c together with the radial $\mathrm{Ti}$ atomic fraction plot (Fig. 6d). The radial integral plot of by a second order polynomial in the region $r \gg r_{\mathrm{TJ}}$ (green dashed line). The constant of this fit reveals a Ti Gibbs TJ excess of $\Gamma_{\mathrm{Ti}}^{\mathrm{TJ}}=1.5 \mathrm{at} / \mathrm{nm}$. The radial integration of Ti atoms divided by the product of the height $(z)$ and the radius of the cylinder $(r)$ inside the TJ region is linear (black dots in Fig. 66). The the knowledge of the previously given data (Ti grain atomic density, Gibbs GB excess, GB width), a Ti Gibbs TJ excess at the $\mathrm{CoSi}_{2}$ TJ is $\Gamma_{\mathrm{Ti}}^{\mathrm{TJ}}=1.7 \mathrm{at} / \mathrm{nm}$.

A positive Ti Gibbs TJ excess value at the $\mathrm{CoSi}_{2}$ TJ is revealed from all three methods confirming the qualitative observation. However, there is a difference in the excess values from the three methods. The value determined by the cumulative profile is the highest $(2.2 \mathrm{at} / \mathrm{nm})$ while the radial integration method in the range $r \gg r_{\mathrm{TJ}}$ delivers the lowest value $(1.5 \mathrm{at} / \mathrm{nm})$ and the radial integration method in the range $r \leq r_{\mathrm{TJ}}$ a value in between $(1.7 \mathrm{at} / \mathrm{nm})$. Approximations and limitations of the three methods, which are partly explained in the section

above, need further discussion for better understanding of the applicability of the methods as well as the meaning of the resulting value itself. 

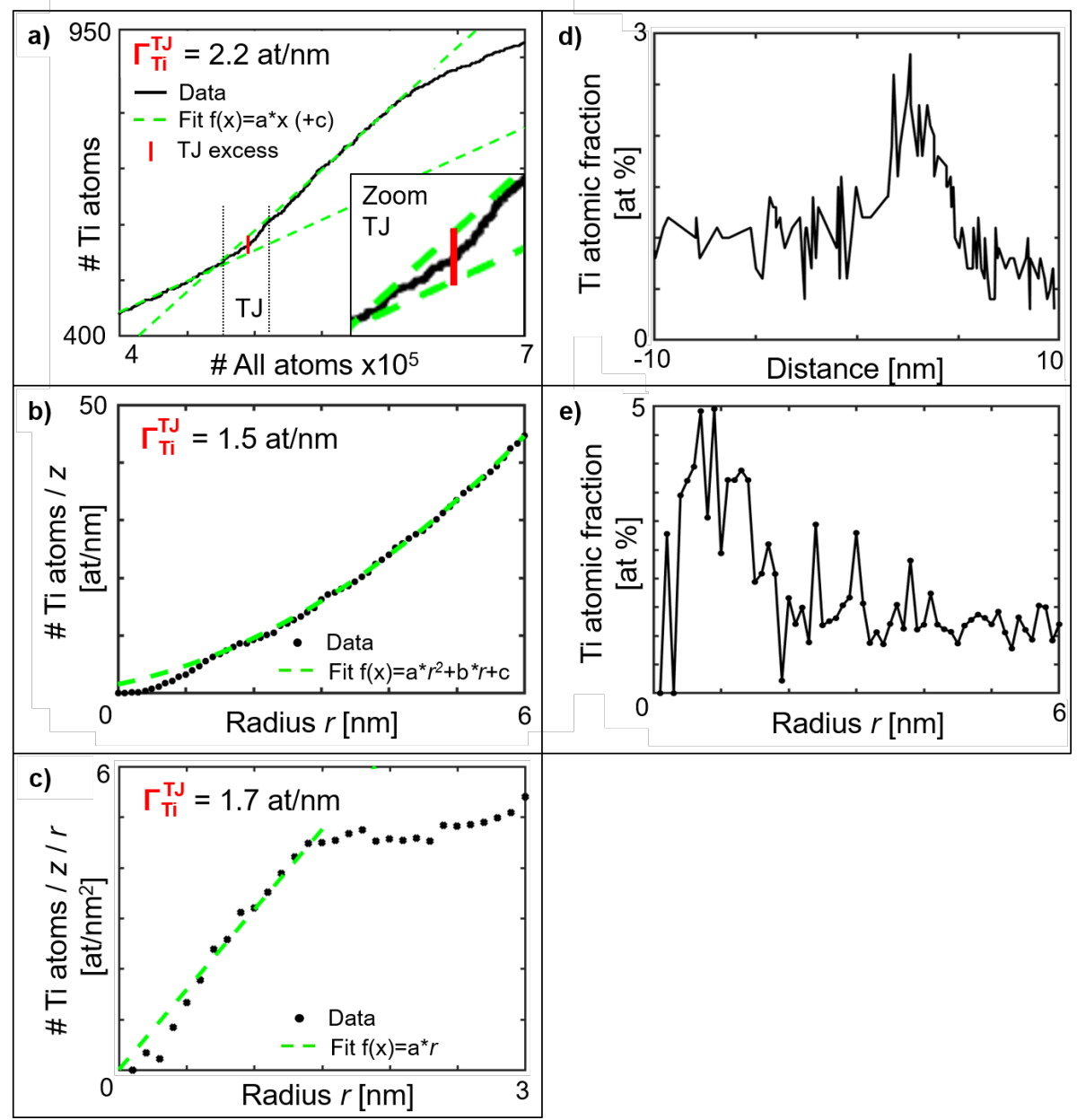

Figure 6: Quantification of $\mathrm{Ti}$ excess at $\mathrm{CoSi}_{2} \mathrm{TJ}$ in experimentally acquired APT volume from Fig. 5a. a) Cumulative profile method. b) Radial integration method in the approach $r \gg r_{\mathrm{TJ}}$. c) Radial integration method in the approach $r \leq r_{\mathrm{TJ}}$. d) Linear 1D Ti atomic fraction plot related to a). e) Radial 1D Ti atomic fraction plot related to b) and c).

\section{Discussion}

Higher concentrations of solutes at TJs have already been reported in literature for solute diffusion [15,19] or segregation [10, 11, but without quantifica-

tion of the Gibbs TJ excess. Our work provides three methods for a quantification of Gibbs TJ excess: one based on a cumulative profile and two on a radial 
integration profile. The advantages and limitations of these methods will now be discussed.

A cumulative profile method in which the number of a specific atom is plot355 ted as a function of the number of all atoms is sensitive to the atomic fraction since it is the slope of such a plot. The radial integration methods that use the number of solutes over a distance are sensitive to the atomic density since they count atoms per volume instead per total number of atoms (slope is proportional to the atomic density). State-of-the-art APT reconstruction procedures 360 can deliver local spatial resolution in the range of sub nanometers resolving crystal lattice planes. However, their precision can suffer from detection efficiency variations or local magnification effects, especially at interfaces. When the detection efficiency varies for different elements, the reconstructed atomic fractions can be affected and thus cumulative profile analysis. On the other 365 hand, local magnification effects can change the reconstructed atomic densities affecting the analysis using the presented radial integration methods. In principle, cumulative profile analysis could also be applied in the radial geometry in order to determine the effects of possible detection efficiency variation and local magnifications. However, there is no direct link to the Gibbs TJ excess in the 370 range $r \gg r_{\text {TJ }}$ when a radial cumulative profile is used instead of a radial integration profile. In the range $r \leq r_{\mathrm{TJ}}$, the atomic fraction of Ti in the TJ region can be determined. It is related to the excess number of atoms at the TJ (Eq. 3). Nevertheless, knowledge about the TJ area is needed among other geometries in order to calculate the Gibbs TJ excess from the atomic fraction. This area is 375 related to $r_{\mathrm{TJ}}^{2}$ and thus again related to an absolute distance (atomic density) in the reconstruction, even to the power of two, making a direct comparison of the effects of artificial variations in the reconstruction of atomic fraction or atomic density very difficult. 
There are other advantages and short comings for each method than being

380 cumulative profile made from a linear integration, there is more freedom in the choice of the investigated region. The geometry of the cuboid which selects the region of investigation can be varied as well as its orientation relative to the GBs allowing to avoid obvious inhomogeneities. High statistics can be reached when the TJ geometry intrinsically necessary. One of the limitations of this method is the high sensitivity of the TJ excess on the choice of a center location between the neighboring regions of constant atomic fractions. Moreover, this method is not appropriate when the close environment of the TJ does not provide regions of constant solute atomic fraction in the chosen cumulative profile analysis. The radial integration method in the range $r \gg r_{\mathrm{TJ}}$ has similar advantages such as good statistics from a large investigated volume and no assumptions on the exact TJ structure are necessary. However, the determination of the TJ excess from the constant of a second order polynomial fit depends highly on the quality is not applicable for inhomogeneous radial solute distributions around the TJ region. The radial integration method in the range $r \leq r_{\mathrm{TJ}}$ is complementary to the previous methods and so are its advantages. A linear fit instead of a second order polynomial is a priori more reliable. In addition, inhomogeneities around However, the assumed geometry of the TJ is important for the result and needs to be representative for the investigated TJ as the assumed homogeneous radial solute distribution inside the TJ region.

While the investigated volume for the cumulative profile analysis can be optimized for best sensitivity, the volumes of the radial integration are fixed by 
definition. The radial integration methods can balance this drawback due to their complementarity requirements on the TJ geometry of the two radial integration analyses $\left(r \gg r_{\mathrm{TJ}}\right.$ : tight GBs and thereby TJ, $r \leq r_{\mathrm{TJ}}$ : wide GBs and thereby large TJ region) allowing to choose the optimal method depending on the present TJ geometry in extreme cases or applying both in medium cases as in our present experimental volume. For all three methods, a correct alignment of the investigating volume with the TJ axis is of high importance. Misalignments can lead to variations in the fitted atomic fractions or atomic densities and thus to deviations in the determined Gibbs TJ excess value.

Furthermore, the dihedral angles have been set in the model volume to about $120^{\circ}$ with the argument of the representation of an equilibrium state. For significant variations of the dihedral angle values from $120^{\circ}$, it is possible that the $\mathrm{TJ}$ region is not as homogeneously filled with GB regions in difference to the schematic visualization in Fig. 17. A highly asymmetric GB overlap can be formed which would question the validity of the TJ volume approximation as a cylinder including the three GB overlap. The validation of this model is of specific importance for the radial integration method in the range $r \leq r_{\mathrm{TJ}}$ (third method), because the atomic fraction can become inhomogeneously (or just not representative) when the defined TJ volume is not homogeneously filled with GB contributions. Furthermore, such an asymmetry can affect strongly the cumulative method (first method) since a choice for the position of the step between the linear fits from bordering regions with constant atomic fraction is necessary. Correlative structural and chemical information at sub Ångström length scale using, for example, high angle annular dark field scanning transmission electron microscopy measurements could give insights to the usability of the proposed model and methods. However, they are limited regarding the possibility to project the structure along the TJ axis. On the other hand, such asymmet- 
ric GB arrangements do not represent equilibrium states. In our experimental volume, the dihedral angles are around $120^{\circ}$ which represent a state close to equilibrium and the usage of the three proposed methods with the geometric assumptions should be justified.

A similar geometry was used in a theoretical study on GB and TJ segregation in a binary Fe-Cr alloy by Eich et al. [20]. Segregation to GBs and TJ was investigated for a bulk concentration ranging from high Fe concentrations to high Cr concentrations. Different temperature and different chemical potentials have been set. Gibbs GB excess values up to the order of $100 \mathrm{at} / \mathrm{nm}^{2}$ were found. For the Gibbs TJ excess, values up to the order of 10 at $/ \mathrm{nm}$ were obtained. The excess values vary depending on the bulk concentration. In our work, the determined experimental Ti Gibbs TJ excess at a $\mathrm{CoSi}_{2}$ TJ is one order of magnitude lower than the maximum Gibbs TJ excess calculated in the Fe-Cr alloy. These lower excess values in our study can explain the high relative variations mentioned for the three presented methods. Reasons for the difference in the order of magnitude between the theoretical study of Eich et al. [20] and our experimental work can be the different material system or different crystallography (misorientation of adjacent GBs, orientation of GB planes and TJ line) of the considered TJs. In future experimental studies, it may be possible to determine the TJ crystallography in addition to the chemistry either by using complementary techniques (such as transmission kikuchi diffraction in scanning electron microscope [41, 42] or diffraction analysis in transmission electron microscope [43, 44]). APT could also provide enough crystallographic information [45, 46] if special acquisition conditions are fulfilled such as: TJ line centered in APT tip along analysis direction, acquisition parameter setting optimized for crystallographic information. This will allow a better quantitative comparison with theoretical calculations on the same material system and the 
same crystallography of the TJ.

Such future studies could bring more light into the question of the nature of the TJ excess which can have different origins such as segregation to structural defects, formation of another phase at the junction or simply geometrical descriptions. A geometric estimation of the Gibbs TJ excess was given in the discussion of Eich et al. 20 (Eq. 18): $\Gamma^{\mathrm{TJ}}=\sqrt{3} / 4 \cdot d_{\mathrm{GB}} \cdot \Gamma^{\mathrm{GB}}$ by considering the overlap of the GB regions (Fig. 1 in 20 ). It is an approximation which bases on the geometry of the adjacent GBs width assuming dihedral angles of $120^{\circ}$. From the match between estimation and calculated values, it was concluded that Gibbs TJ excess can be described as a geometric effect of the finite width of the GB zones and a result of the GB overlapping zones [20].

In our work, such an GB overlap depended TJ excess can be estimated by the multiplication of the projected overlap areas (Eq. A.3 in Appendix A with the average GB Ti atomic densities (Eq. 21. For our experimental volume it gives a TJ excess of about $\Gamma_{\mathrm{Ti}}^{\mathrm{TJ} \text {,overlap }}=0.9 \mathrm{at} / \mathrm{nm}$. This value is lower than the determined excess at the TJ suggesting that a geometrical explanation from GB overlap cannot account for the full value of the TJ excess. This opens the question for more precise explanations of TJ excess origins than confinement of finite GB widths.

Another approach which is commonly used for the determination of excess is the application of proximity histograms (proxigrams [47]). Thereby, interfaces are created by the calculation of local concentrations (atomic fractions) and connecting a net (surface) for an isoconcentration value (similar for atomic density with isodensity value). The advantage of such an approach is that the shape of the interfaces can be complex [48. For segregation at GBs or interphase boundaries, it is appropriate to develop procedures which map interfacial excess and surface concentrations [49]. Reliable reconstruction of atomic density 
or atomic fraction is still crucial. Improvements on this approach were recently reached by subdividing the interface for more accurate excess analysis in terms of spatial distribution of segregates and automatization of analysis [50. Even though a TJ does not represent an interface in a classical sense, the proxigram approach may provide details in TJs about local distributions of segregation in correlation with structures or structural defects. However, the definition of useful isoconcentration interfaces can be very delicate. Furthermore, such analyses are limited in their representativity since they characterize subregions of the TJ while the Gibbs TJ excess gives an average value for the characterized TJ region. The methods presented in our work are convenient approaches to quantify a Gibbs TJ excess. This value is of high interest for various applications [51. It is also of great interest from a fundamental point of view in relationship with crystallographic parameters like misorientations of the adjacent grains and relative orientation of the TJ axis as well as for different materials or different material processing [52].

Ongoing work aims for improvements of the presented methods. In the linear integration method higher reproducibility and reliability could be achieved by finding automatically optimal fit regions as well as the center of the TJ region similarly to optimization on interface segregation quantifications [50. For the radial integration methods, improvements on even more reliable reconstruction of atomic densities and thereby distances in the tomographic reconstruction, especially at interfaces, are essential.

\section{Conclusion}

Three methods have been presented to determine the Gibbs TJ excess in $3 \mathrm{D}$ volumes obtained by APT that has an atomic resolution and the capability to count single atoms. Their functionality has been demonstrated on simulated 
model volumes with well-defined geometries and concentrations of GBs and TJ. These methods based on cumulative profile and radial integration of solute 515 atoms have been compared.

An experimental APT volume of $\mathrm{CoSi}_{2}$ containing $3 \mathrm{GBs}$ with Ti segregation joining together in a TJ has been analyzed. Ti excess at the TJ can be visualized with 2D Ti atomic density or atomic fraction maps. Applying the three methods, the Ti Gibbs TJ excess at the $\mathrm{CoSi}_{2}$ TJ is quantified revealing positive excess values. To conclude, there is Gibbs TJ excess of $\mathrm{Ti}$ at the investigated $\mathrm{CoSi}_{2}$ TJ which influences its structure and properties and further the properties of the entire thin film.

The demonstrated quantification of Gibbs TJ excess opens the door for a more complete characterization of poly crystalline materials. It provides a more ${ }_{525}$ accurate correlation between chemical characterization at the atomic scale and materials properties. For such a correlation, a systematic study of selected TJ would be needed since poly crystalline materials, depending on their texture, consists of various TJs that can have different influence on the properties.

\section{Acknowledgment}

530 This work was supported by the French government through the program "Investissements d'Avenir A*MIDEX" (Project APODISE, no. ANR-11-IDEX0001-02) managed by the National Agency for Research (ANR). The authors would like to thank Maxime Bertoglio for assistance with sample preparation and Marion Descoins for assistance with APT tip preparation and APT measurements.

\section{Conflict of interest}

The authors declare that they have no conflict of interest. 


\section{References}

[1] A. H. King. Interface Science 7 (1999) 251-271.

${ }_{540} \quad$ [2] J. Taylor. Interface Science 7 (1999) 243-249.

[3] A. Caro and H. Van Swygenhoven. Physical Review B 63 (2001) 134101.

[4] G. Gottstein, L. S. Shvindlerman and B. Zhao. Scripta Materialia 62 (2010) 914-917.

[5] P. Fortier, G. Palumbo, G. D. Bruce et al. Scripta Metallurgica et Materiala 25 (1991) 177-182.

[6] B. Zhao, J. C. Verhasselt, L. S. Shvindlerman et al. Acta Materialia 58 (2010) 5646-5653.

[7] S. G. Srinivasan, J. W. Cahn, H. Jónsson et al. Acta Materialia 47 (1999) $2821-2829$.

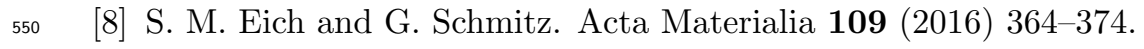

[9] A. H. King. Materials Science and Technology 23 (2007) 505-508.

[10] K. Yin, A. H. King and T. Hsieh. Microscopy and Microanalysis 3 (1997) 417-422.

[11] J. Tian and Y. L. Chiu. Materials Characterization 148 (2019) 156-161.

555 [12] H. Kim, Y. Xuan, P. D. Ye et al. Acta Materialia 57 (2009) 3662-3670.

[13] B. Gault, M. P. Moody, J. M. Cairney et al. Springer, 2012.

[14] W. Lefebvre, F. Vurpillot and X. Sauvage. Elsevier Science, 2016.

[15] M. R. Chellali, Z. Balogh, L. Zheng et al. Scripta Materialia 65 (2011) 343-346. 
[16] M. R. Chellali, Z. Balogh, H. Bouchikhaoui et al. Nano Letters 12 (2012) $3448-3454$.

[17] M. R. Chellali, Z. Balogh and G. Schmitz. Ultramicroscopy 132 (2013) $164-170$.

[18] P. Stender, Z. Balogh and G. Schmitz. Physical Review B - Condensed

[19] P. Stender, Z. Balogh and G. Schmitz. Ultramicroscopy 111 (2011) 524529.

[20] S. M. Eich and G. Schmitz. Acta Materialia 147 (2018) 350-364.

[21] J. W. Gibbs. London, Longmans, Green, \& co., 1928.

570 [22] B. W. Krakauer and D. N. Seidman. Physical Review B 48 (1993) 67246727.

[23] S. P. Murarka. Intermetallics 3 (1995) 173-186.

[24] S. S. Lau, J. W. Mayer and K. N. Tu. Journal of Applied Physics 49 (1978) 4005-4010.

575 [25] F. M. D'Heurle and C. S. Petersson. Thin Solid Films 128 (1985) 283-297.

[26] M. L. A. Dass, D. B. Fraser and C.-S. Wei. Applied Physics Letters 58 (1991) 1308.

[27] S. L. Hsia, T. Y. Tan, P. Smith et al. Journal of Applied Physics 70 (1991) $7579-7587$.

${ }_{580}$ [28] P. Liu, B.-Z. Li, Z. Sun et al. Journal of Applied Physics 74 (1993) 17001706. 
[29] F. Hong, G. A. Rozgonyi and B. K. Patnaik. Applied Physics Letters 64 (1994) 2241-2243.

[30] T. I. Selinder, T. A. Roberts, D. J. Miller et al. Journal of Applied Physics 77 (1995) 6730.

[31] C. Detavernier, R. L. Van Meirhaeghe, F. Cardon et al. Applied Physics Letters 77 (2000) 3170-3172.

[32] C. Detavernier, R. L. Van Meirhaeghe, F. Cardon et al. Microelectronic Engineering 50 (2000) 125-132.

590 [33] C. Detavernier, R. L. Van Meirhaeghe, W. Vandervorst et al. Microelectronic Engineering 71 (2004) 252-261.

[34] H. Zschiesche, A. Charaï, D. Mangelinck et al. Microelectronic Engineering 203-204 (2019) 1-5.

[35] M. K. Miller, K. F. Russell, K. Thompson et al. Microscopy and Microanalysis 13 (2007) 428-436.

[36] P. J. Felfer, T. Alam, S. P. Ringer et al. Microscopy Research and Technique 75 (2012) 484-491.

[37] P. Bas, A. Bostel, B. Deconihout et al. Applied Surface Science 87-88 (1995) 298-304.

[38] F. Vurpillot, B. Gault, B. P. Geiser et al. Ultramicroscopy 132 (2013) 19-30.

[39] P. J. Warren, A. Cerezo and G. D. W. Smith. Ultramicroscopy 73 (1998) 261-266.

[40] B. M. Jenkins, F. Danoix, M. Gouné et al. ArXiv ID: 1806.03851 (2018). 
[41] G. C. Sneddon, P. W. Trimby and J. M. Cairney. Materials Science and Engineering R: Reports 110 (2016) 1-12.

[42] T. Schwarz, G. Stechmann, B. Gault et al. Progress in Photovoltaics: Research and Applications 26 (2017) 196-204.

[43] E. F. Rauch, J. Portillo, S. Nicolopoulos et al. Zeitschrift fur Kristallographie 225 (2010) 103-109.

[44] S. Zaefferer. Crystal Research and Technology 46 (2011) 607-628.

[45] M. P. Moody, F. Tang, B. Gault et al. Ultramicroscopy 111 (2011) 493-499.

[46] A. J. Breen, K. Babinsky, A. C. Day et al. Microscopy and Microanalysis 23 (2017) 279-290.

${ }_{615}^{6}$ [47] O. C. Hellman, J. A. Vandenbroucke, J. Rüsing et al. Microscopy and Microanalysis 6 (2000) 437-444.

[48] O. C. Hellman and D. N. Seidman. Materials Science and Engineering A 327 (2002) 24-28.

[49] P. Felfer, B. Scherrer, J. Demeulemeester et al. Ultramicroscopy 159 (2015) $438-444$.

[50] Z. Peng, Y. Lu, C. Hatzoglou et al. Microscopy and Microanalysis 25 (2019) 389-400.

[51] B. Zhao, G. Gottstein and L. S. Shvindlerman. Acta Materialia 59 (2011) 3510-3518.

${ }_{625}$ [52] D. Mattissen, D. A. Molodov, L. S. Shvindlerman et al. Acta Materialia 53 (2005) 2049-2057. 


\section{Appendix A. Geometrical considerations}

\section{$T J$ radius $\left(r_{T J}\right)$ and $G B$ overlap determination}

Fig. A.7 is an visualization of the TJ geometry similar to Fig. 1 with only two GBs that have identical GB width $\left(d_{\mathrm{GB}, 1}=d_{\mathrm{GB}, 2}=d_{\mathrm{GB}}\right)$ for simplicity. The projected GB1 appears in a green frame while GB2 is framed in orange. The center of their junction represents the TJ. The frame of the TJ cylinder projection is drawn as a blue circle. The radius of the TJ cylinder $\left(r_{\mathrm{TJ}}\right)$ was defined as the radius of the circle that includes all occurring GB overlap at the TJ. The overlapping area is a tetragon and marked in yellow. The illustration shows that $r_{\mathrm{TJ}}$ is thereby defined as the diagonal of the overlapping area of the adjacent GBs in the considered case. The angle of the diagonal of the GB overlap area can be calculated to

$$
\epsilon=\frac{\pi-\gamma}{2}
$$

and with the knowledge of the GB width, the radius of the TJ is

$$
r_{\mathrm{TJ}}=\frac{d_{\mathrm{GB}}}{2} \cdot \frac{1}{\cos (\epsilon)} .
$$

Furthermore, the overlapping area of the adjacent GBs is

$$
\begin{aligned}
S_{12} & =\frac{d_{\mathrm{GB}}}{2} \cdot \overline{C B} \\
\overline{C B} & =\frac{d_{\mathrm{GB}}}{2} \cdot \tan (\epsilon) \\
S_{12} & =\left(\frac{d_{\mathrm{GB}}}{2}\right) \cdot \tan (\epsilon)
\end{aligned}
$$

\section{GB fraction in $T J$}

The volume fraction of a single GB in the TJ is related to the area of the GB slice in the TJ cylinder projected along the TJ axis (Fig. A.8). It is composed 


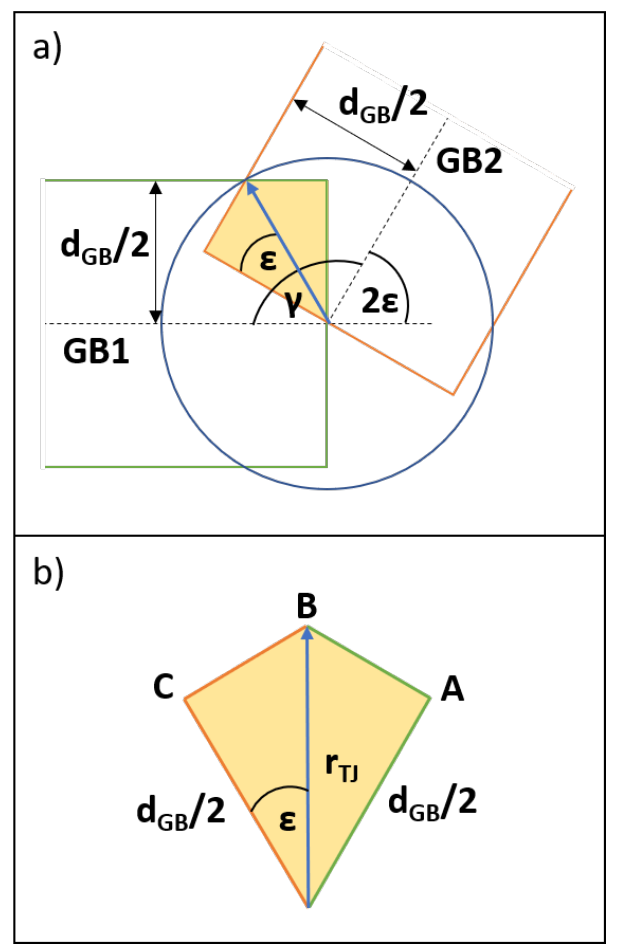

Figure A.7: Schema of GB overlap in TJ region for visualization of TJ radius and its calculation as well as calculating the overlap area.

of the horizontally dashed rectangular area $A_{\text {rect }}$ and the vertically dashed arc area $A_{\text {arc }}$.

$$
\begin{aligned}
A_{\text {rect }} & =\sqrt{r_{\mathrm{TJ}}^{2}-\frac{d_{\mathrm{GB}}^{2}}{4}} \cdot d_{\mathrm{GB}} \\
A_{\mathrm{arc}} & =\pi r_{\mathrm{TJ}}^{2} \cdot \frac{2 \arcsin \left(\frac{d_{\mathrm{GB}} / 2}{r_{\mathrm{TJ}}}\right)}{360^{\circ}}-\frac{A_{\text {rect }}}{2}
\end{aligned}
$$

The so calculated projected GB area requires additional correction when all three GBs are considered in the TJ because of GB overlap (Fig. 1, Fig. A.7): The half of each of the GB overlap rectangles to the adjacent GBs needs to be 


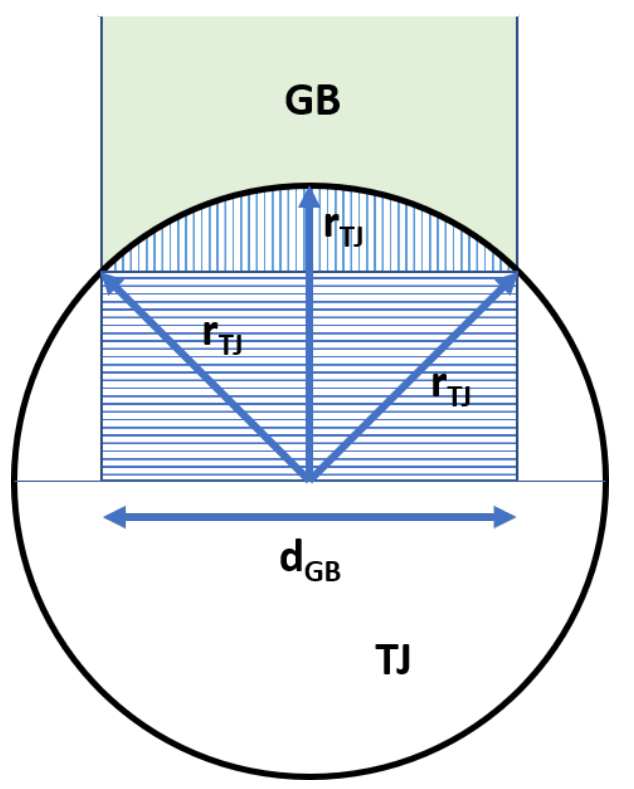

Figure A.8: Visualization of single GB fraction in TJ cylinder (no GB overlap). The area of a single GB in the TJ projected along the TJ axis is the sum of the horizontally stripped rectangular area $A_{\text {rect }}$ and the vertically stripped arc area $A_{\text {arc }}$.

subtracted

$$
A_{\mathrm{GB}, \mathrm{i}}^{\mathrm{TJ}}=A_{\text {rect, } \mathrm{i}}+A_{\mathrm{arc}, \mathrm{i}}-\Sigma_{j \neq i} S_{\mathrm{ij}} / 2 .
$$

Knowing this projected GB area in the TJ, the volume fraction is calculated by the ratio to the base area of the TJ cylinder

$$
f_{\mathrm{GB}, \mathrm{i}}^{\mathrm{TJ}}=\frac{A_{\mathrm{GB}, \mathrm{i}}^{\mathrm{TJ}}}{\pi r_{\mathrm{TJ}}^{2}} .
$$

In general, $d_{\mathrm{GB}}$ is not necessarily the same for all three GBs and $r_{\mathrm{TJ}}$ depends on both the GB widths and the dihedral angles of the adjacent GBs. The overlapping areas are than more complex and three cases can be distinguished.

${ }_{655}$ In the case that the widths of the GBs are different and $d_{\mathrm{GB}, \mathrm{i}}>d_{\mathrm{GB}, \mathrm{j}}$ and 
$d_{\mathrm{GB}, \mathrm{i}} \leq\left|d_{\mathrm{GB}, \mathrm{j}} / \cos (\gamma)\right|$ is fulfilled, the equations for $S_{\mathrm{ij}}$ and $r_{\mathrm{TJ}}$ become:

$$
\begin{aligned}
S_{\mathrm{ij}}= & \frac{d_{\mathrm{GB}, \mathrm{i}} \cdot d_{\mathrm{GB}, \mathrm{j}}}{4} \sin (\gamma)+\frac{d_{\mathrm{GB}, \mathrm{j}}^{2}}{8} \sin (\gamma) \cos (\gamma) \\
& +\frac{1}{2}\left(\frac{d_{\mathrm{GB}, \mathrm{i}}}{2}+\frac{d_{\mathrm{GB}, \mathrm{j}}}{2} \cos (\gamma)\right)^{2} \arctan (\gamma) \\
r_{\mathrm{TJ}}= & \sqrt{\left(d_{\mathrm{GB}, \mathrm{i}} / 2\right)^{2}+\left(d_{\mathrm{GB}, \mathrm{i}} /(2 \cdot \tan (\gamma))+d_{\mathrm{GB}, \mathrm{j}} /(2 \cdot \sin (\gamma))\right)^{2}} .
\end{aligned}
$$

When $d_{\mathrm{GB}, \mathrm{i}}<\left|d_{\mathrm{GB}, \mathrm{j}} / \cos (\gamma)\right|$ and $\gamma>\pi / 2$, Eq. A.7 and A.8 reduce to

$$
\begin{aligned}
S_{\mathrm{ij}} & =\frac{1}{2}\left(\frac{d_{\mathrm{GB}, \mathrm{j}}}{2}\right)^{2}|\tan (\gamma)| \\
r_{\mathrm{TJ}} & =\left|\frac{d_{\mathrm{GB}, \mathrm{j}}}{2 \cdot \cos (\gamma)}\right| .
\end{aligned}
$$

